\begin{tabular}{|c|l|}
\hline Title & Global Solvability of Constrained Singular Diffusion Equation A ssociated with Essential V ariation \\
\hline Author(s) & Giga, Y oshikazu; Kuroda, Hirotoshi; Yamazaki, Noriaki \\
\hline Citation & Hokkaido University Preprint Series in Mathematics, 778, 1-10 \\
\hline Issue Date & 2006 \\
\hline DOI & 10.14943/83928 \\
\hline Doc URL & http://hdl.handle.net/2115/69586 \\
\hline Type & bulletin (article) \\
\hline File Information & pre778.pdf \\
\hline
\end{tabular}

Instructions for use 


\title{
Global Solvability of Constrained Singular Diffusion Equation Associated with Essential Variation
}

\author{
Yoshikazu GIGA, Hirotoshi KURODA, and Noriaki YAMAZAKI
}

\begin{abstract}
We consider a gradient flow system of total variation with constraint. Our system is often used in the color image processing to remove a noise from picture. In particular, we want to preserve the sharp edges of picture and color chromaticity. Therefore, the values of solutions to our model is constrained in some fixed compact Riemannian manifold. By this reason, it is very difficult to analyze such a problem, mathematically. The main object of this paper is to show the global solvability of a constrained singular diffusion equation associated with total variation. In fact, by using abstract convergence theory of convex functions, we shall prove the existence of solutions to our models with piecewise constant initial and boundary data.
\end{abstract}

\section{Introduction}

We consider a constrained singular diffusion equation associated with total variation as follows:

$$
u^{\prime}=-\pi_{u}\left(-\operatorname{div}\left(\frac{\nabla u}{|\nabla u|}\right)\right) \quad \text { in }(0, T) \times \Omega,
$$

where $u^{\prime}(t):=\frac{d}{d t} u(t), 0<T<+\infty$ and $\Omega$ is a bounded domain in $\mathbb{R}^{2}$ with boundary $\Gamma$. Let $S^{n-1}$ be the unit sphere in $\mathbb{R}^{n}(n \geq 1)$, i.e.

$$
S^{n-1}:=\left\{w \in \mathbb{R}^{n} ;|w|=1\right\} .
$$

For each element $u \in S^{n-1}$, let $\pi_{u}: \mathbb{R}^{n} \rightarrow T_{u} S^{n-1}$ be an orthogonal projection from $\mathbb{R}^{n}=T_{u} \mathbb{R}^{n}$ to tangent space $T_{u} S^{n-1}$ of $S^{n-1}$ at $u$.

The motivation of this paper is the color image processing. The constrained singular diffusion equation (1.1) was proposed by Tang-Sapiro-Caselles [22, 23] in

Received by the editors December 1, 2001.

1991 Mathematics Subject Classification. Primary 35K55, 68U10; Secondary 47J35.

Key words and phrases. Singular diffusion, total variation, color image processing, subdifferential. 
order to remove a noise from the chromaticity of the initial image preseving the sharp edges of picture and color chromaticity.

For the gray image processing, there is a vast literature. For instance, we refer to $[1,2,6,19,20]$. In the simplest model of the gray image processing, the Gaussian filter was used for a grey-level function. Namely, for a given initial greylevel function $u_{0}$, we solve the heat equation

$$
u^{\prime}=\Delta u \quad \text { in }(0, T) \times \Omega
$$

to get a denoised grey-level function $u(t, \cdot)$ at scale $t$. However, this way has a drawback since all characteristic function is mollified and a sharp contrast become ambiguous. In order to keep the sharp edges, one use a (unconstrained) singular diffusion equation governed by total variation flow

$$
\left\{\begin{array}{cll}
u^{\prime}=\operatorname{div}\left(\frac{\nabla u}{|\nabla u|}\right) & \text { in } & (0, T) \times \Omega, \\
u=g(x) & \text { on } & (0, T) \times \Gamma, \\
u(0, \cdot)=u_{0} & \text { in } \quad & \Omega,
\end{array}\right.
$$

where $g$ and $u_{0}$ are given data. Then, the grey-level function is not mollified, and a Heaviside type function is a stationary solution to (1.2). Since (1.2) is the gradient system, we easily get the results on existence and uniqueness of solutions. In fact, we can define the energy functional $\psi$ on $L^{2}(\Omega)$ by

$$
\psi(u)=\left\{\begin{array}{cl}
\int_{\Omega}|\nabla \widetilde{u}| & \text { if } \widetilde{u} \in B V(\Omega), \\
+\infty & \text { otherwise. }
\end{array}\right.
$$

Here $\widetilde{u}$ is the extension of $u \in L^{2}(\Omega)$ to $\mathbb{R}^{2}$ such that $\widetilde{u}(x)=\widetilde{g}(x)$ for $x \in \mathbb{R}^{2} \backslash$ $\Omega$, where $\widetilde{g}$ is a Lipschitz extension of the boundary data $g$ to $\mathbb{R}^{2}$. Then, $\psi$ is proper, lower semi-continuous and convex on $L^{2}(\Omega)$. Moreover, the (unconstrained) gradient system (1.2) can be reformulated as in the abstract form:

$$
u^{\prime}(t) \in-\partial \psi(u(t)) \quad \text { in } L^{2}(\Omega), \quad \text { for } t>0 .
$$

Thus, by applying the general theory established by Brézis [5] and Kōmura [16], we can get the solution to (1.2). For another detailed analysis, we refer to [1, 2], for instance. The (unconstrained) singular diffusion equation is also important to describe nonlinear physical phenomena (cf. $[8,10,14,15,21,24]$ ). For instance, Shirakawa-Kimura [21] studied Allen-Cahn type equation with the total variation functional as the interfacial energy.

In this paper we discuss the global existence of solution $u:[0, T) \times \Omega \rightarrow$ $S^{n-1} \subset \mathbb{R}^{n}$ to the following Dirichlet problem

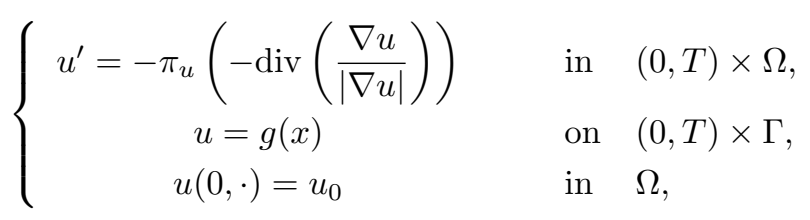


where $g$ and $u_{0}$ are given data which are maps from $\Omega$ to $S^{n-1}$. In 2003, GigaKobayashi [10] considered the problem (1.5) in the one-dimensional case. Then, they showed that for each piecewise constant initial data $u_{0}$ on $\Omega$, there is a unique global solution $u$ on $[0, \infty)$ such that $u(t)$ is a piecewise constant on $\Omega$. Moreover, they studied the stationary problem in the case when the manifold is the unit circle $S^{1}$ in $\mathbb{R}^{2}$. In 2004, Giga-Kashima-Yamazaki [9] studied (1.1) in the $n$-dimensional torus domain $\Omega:=\mathbb{T}^{n}=\prod_{i=1}^{n}\left(\mathbb{R} / \omega_{i} \mathbb{Z}\right)$ for given $\omega_{i}>0(i=1,2, \cdots, n)$. Assuming that the initial data $u_{0}$ is (sufficiently) small in some sense, they [9] constructed the local solution to (1.1) in the torus domain $\mathbb{T}^{n}$ as the limit of solutions to $p$-harmonic map flow equations with $p>1$

$$
u^{\prime}=-\pi_{u}\left(-\operatorname{div}\left(|\nabla u|^{p-2} \nabla u\right)\right) \quad \text { in }(0, T) \times \mathbb{T}^{n}
$$

by passing to the limit of $p \rightarrow 1$. In 2005, Giga-Kuroda-Yamazaki [12] proved the global existence of solution to a discretized version of (1.1) with Neumann boundary condition by restricting a class of mappings into that of piecewise constant mappings.

The main object of this paper is to show a global solvability of (a discretized version of) Dirichlet problem (1.5) by using the idea of $[9,10,12]$. Namely, for each piecewise constant initial and boundary data we find the piecewise constant solution $u(t)$ to $(1.5)$ on $\Omega$. Then, the problem is reduced to a system of ordinary differential equations unless two different values merges. This is the key point and idea in order to construct the global solution to the discretized Dirichlet problem (1.5). Of course, merging may occur, so, it is very difficult to study the detailed dynamics in 2-dimensional case. Different from one dimensional problem, our approach may not correspond to a solution of an original problem with a piecewise constant initial data. Such a difficulty is also observed in the unconstrained problem of crystalline flow [4] and [8], for instance.

The plan of this paper is as follows. In Section 2, we reformulate the problem (1.5) as in the evolution equation in some real Hilbert space by using subdifferential of convex functional. Then, we mention main result (Theorem 2.3) in this paper, which is concerned with the global existence of solution. In Section 3, we recall the convergence theorem established in [9]. In Section 4, we consider the approximating problem to (1.5). In the final Section 5 we give the proof of Theorem 2.3.

\section{Notation}

Throughout this paper, let $\Omega$ be a bounded domain in $\mathbb{R}^{2}$ with boundary $\Gamma$. We denote by $L^{2}\left(\Omega ; \mathbb{R}^{n}\right)$ the space of $\mathbb{R}^{n}$-valued square integrable functions. For the unit sphere $S^{n-1}$ in $\mathbb{R}^{n}(1 \leq n<+\infty)$, let $L^{2}\left(\Omega ; S^{n-1}\right)$ be the closed subset of $L^{2}\left(\Omega ; \mathbb{R}^{n}\right)$ of the form

$$
L^{2}\left(\Omega ; S^{n-1}\right):=\left\{v \in L^{2}\left(\Omega ; \mathbb{R}^{n}\right) ; v(x) \in S^{n-1} \text { a.e. } x \in \Omega\right\} .
$$

Let $H$ be a real Hilbert space with the inner product $\langle\cdot, \cdot\rangle$, and $\varphi: H \rightarrow$ $(-\infty,+\infty]$ be a proper (i.e., not identically equal to infinity), l.s.c. (lower semicontinuous) and convex function on $H$. Then, we denote by $\partial \varphi$ the subdifferential 
of $\varphi$, which is defined by the set

$$
\partial \varphi(u)=\{f \in H \mid \varphi(u+h)-\varphi(u) \geq\langle f, h\rangle \text { for any } h \in H\} .
$$

For basic properties of subdifferential, we refer to the monograph by Brézis [5].

\section{Subdifferential formulation and main theorem}

We begin with the definition of rectangular decompositions of $\Omega$.

Definition 2.1 (Rectangular decomposition). For the bounded domain $\Omega$ in $\mathbb{R}^{2}$, let $\mathcal{C}$ be a rectangular decomposition of $\mathbb{R}^{2}$ so that $\mathcal{C}:=\left\{R_{j}\right\}_{j \in \Lambda}$ is a disjoint family of open rectangles $R_{j}=\left(a_{j}, b_{j}\right) \times\left(c_{j}, d_{j}\right)$ which covers $\mathbb{R}^{2}$ expect a Lebesgue measure zero set. Then, we define a decomposition $\Delta$ of $\Omega$ associated with $\mathcal{C}$ by

$$
\Delta:=\left\{\Omega_{i}\right\}_{i \in I} \text { with } \Omega_{i}=R_{i} \cap \Omega, I=\left\{i \in \Lambda ; \Omega_{i} \neq \emptyset\right\} .
$$

Note that $I$ is a finite index set, since $\Omega$ is a bounded domain.

Throughout this paper we fix the family $\Delta=\left\{\Omega_{i}\right\}_{i \in I}$. Then, let $H_{\Delta}$ be the set of all $\mathbb{R}^{n}$-valued step functions on $\bigcup_{i \in I} \Omega_{i}$, i.e.

$$
H_{\Delta}=\left\{\sum_{i \in I} a_{i} \chi_{\Omega_{i}} ; a_{i} \in \mathbb{R}^{n}\right\},
$$

where $\chi_{\Omega_{i}}$ is the characteristic function on $\Omega_{i}$. We easily see that $H_{\Delta}$ is the subset of $L^{2}\left(\Omega ; \mathbb{R}^{n}\right)$, and the total variation of $u \in H_{\Delta}$ is given by this form

$$
\int_{\Omega}|\nabla u|=\sum_{i<j} c_{i j}\left|a_{i}-a_{j}\right| \quad \text { if } u \in H_{\Delta}
$$

which is also called a essential variation of $u$. Here, we set $c_{i j}=\mathcal{H}^{1}\left(\partial \Omega_{i} \cap \partial \Omega_{j}\right)$, where $\mathcal{H}^{1}$ is the Hausdorff measure and $\partial \Omega_{i}$ is the boundary of $\Omega_{i}$. More precisely, $c_{i j}$ implies a length of $\partial \Omega_{i} \cap \partial \Omega_{j}$. For the precise definition and basic properties of total variation, see monographs by Evans-Gariepy [7] or Giusti [13], for instance.

Now, by the similar argument in the gray image processing (1.2)-(1.4), we reformulate the problem (1.5) as in some evolution equation. To do so, let us define two functions on real Hilbert spaces. For given boundary data $g \in H_{\Delta}$, we put

$$
\varphi_{\Delta}(u)=\left\{\begin{array}{cl}
\int_{\Omega}|\nabla u| & \text { if } u \in H_{\Delta} \text { with } u(x)=g(x) \text { for } x \in \Gamma \\
+\infty & \text { otherwise. }
\end{array}\right.
$$

Then, from [7] or [13] it follows that $\varphi_{\Delta}$ is the proper, l.s.c. and convex function on $L^{2}\left(\Omega ; \mathbb{R}^{n}\right)$. Also, we can define the proper, l.s.c. and convex function $\Phi_{\Delta}^{T}$ on $L^{2}\left(0, T ; L^{2}\left(\Omega ; \mathbb{R}^{n}\right)\right)$ by the form (cf. [9, Proposition 2.1$]$ )

$$
\Phi_{\Delta}^{T}(u)=\int_{0}^{T} \varphi_{\Delta}(u(t)) d t \quad \text { for all } u \in L^{2}\left(0, T ; L^{2}\left(\Omega ; \mathbb{R}^{n}\right)\right)
$$


Next, For each $h \in L^{2}\left(0, T ; L^{2}\left(\Omega ; S^{n-1}\right)\right)$ we define a map $P_{h}(\cdot)$ from $L^{2}(0, T$ $\left.; L^{2}\left(\Omega ; \mathbb{R}^{n}\right)\right)$ to $L^{2}\left(0, T ; L^{2}\left(\Omega ; \mathbb{R}^{n}\right)\right)$ by

$$
P_{h}(f)(t, x):=\pi_{h(t, x)}(f(t, x)) \quad \text { for a.e. }(t, x) \in[0, T] \times \Omega
$$

for any $f \in L^{2}\left(0, T ; L^{2}\left(\Omega ; \mathbb{R}^{n}\right)\right)$.

By using these notations as above, we easily see that the problem (1.5) can be reformulated as in the following form:

$$
\begin{cases}u^{\prime} \in-P_{u}\left(\partial \Phi_{\Delta}^{T}(u)\right) & \text { in } L^{2}\left(0, T ; L^{2}\left(\Omega ; \mathbb{R}^{n}\right)\right), \\ \left.u\right|_{t=0}=u_{0} & \text { in } \Omega .\end{cases}
$$

Now, let us give the definition of a solution to (2.4) (i.e. (1.5)).

Definition 2.2. Let $0<T<+\infty$. For given data $g, u_{0} \in H_{\Delta}$, a function $u: \Omega \times$ $[0, T] \rightarrow \mathbb{R}^{n}$ is called a solution of (2.4) (i.e. (1.5)), if $u \in L^{2}\left(0, T ; L^{2}\left(\Omega ; S^{n-1}\right)\right) \cap$ $C\left([0, T], L^{2}\left(\Omega ; \mathbb{R}^{n}\right)\right), u_{t} \in L^{2}\left(0, T ; L^{2}\left(\Omega ; \mathbb{R}^{n}\right)\right)$ and (2.4) holds.

Now, let us mention our main result in this paper, which is concerned with the global existence of a solution to (2.4) (i.e. (1.5)).

Theorem 2.3. Suppose the initial and boundary data $u_{0}, g \in H_{\Delta}$ with $u_{0}, g \in$ $L^{2}\left(\Omega ; S^{n-1}\right)$. Then, for any time $T>0$ there exists at least one solution $u$ on $[0, T]$ to the problem (2.4), i.e. (1.5).

Note that we cannot apply the general theory (cf. $[5,16])$ to the problem (2.4), because of the projection $P_{u}$. Hence, in order to prove Theorem 2.3, we consider the approximating problem of (1.5), and apply the abstract convergence theorem established in [9].

\section{Abstract convergence theory}

In this section, we recall the abstract convergence theory in [9]. We begin with the notion of Graph-convergence for multi-valued operators on a real Hilbert space $H$.

Definition 3.1 (e.g. [3]). For (multi-valued) operators $A_{n}(n=1,2, \cdots)$ and $A$ on a real Hilbert space $H$, we say that $A_{n}$ converges to $A$ in the sense of Graph as $n \rightarrow+\infty$, if for any $(u, v) \in \operatorname{Graph}(A)$ there exists $\left(u_{n}, v_{n}\right) \in \operatorname{Graph}\left(A_{n}\right)$ such that $u_{n} \rightarrow u$ and $v_{n} \rightarrow v$ strongly in $H$ as $n \rightarrow+\infty$.

Example. (cf. [3] or [9, Appendix]). Let $\psi, \psi_{n}(n=1,2, \cdots)$ be proper, l.s.c. and convex functions on $H$. Assume that $\psi_{n}$ converges to $\psi$ on $H$ as $n \rightarrow+\infty$ in the sense of Mosco [18], namely, the following two conditions are satisfied:

(i) For any subsequence $\left\{\psi_{n_{k}}\right\} \subset\left\{\psi_{n}\right\}$, if $z_{k} \rightarrow z$ weakly in $H$ as $k \rightarrow+\infty$, then, $\liminf _{k \rightarrow+\infty} \psi_{n_{k}}\left(z_{k}\right) \geq \psi(z)$.

(ii) For any $z \in D(\psi)=\{z \in H \mid \psi(z)<+\infty\}$, there is a sequence $\left\{z_{n}\right\}$ in $H$ such that $z_{n} \rightarrow z$ in $H$ and $\psi_{n}\left(z_{n}\right) \rightarrow \psi(z)$ as $n \rightarrow+\infty$.

Then, $\partial \psi_{n}$ converges to $\partial \psi$ on $H$ in the sense of Graph as $n \rightarrow+\infty$. 
Next, let us introduce the class $\mathcal{L}(K)$ of the operator $B(\cdot)(\cdot): L^{2}(0, T ; G) \times$ $L^{2}(0, T ; H) \rightarrow L^{2}(0, T ; H)$, where $G$ is a non-empty closed subset of $H$ and $L^{2}(0, T ; G)$ is a closed subset of $L^{2}(0, T ; H)$ of the form

$$
L^{2}(0, T ; G):=\left\{u \in L^{2}(0, T ; H) ; u(t) \in G \text { a.e. } t \in[0, T]\right\} .
$$

Definition 3.2 (cf. [9, Section 3]). We denote by $B \in \mathcal{L}(K)$ the set of all operator $B(\cdot)(\cdot): L^{2}(0, T ; G) \times L^{2}(0, T ; H) \rightarrow L^{2}(0, T ; H)$ satisfying the following three conditions:

(i) For any $u \in L^{2}(0, T ; G), B(u)(\cdot)$ is a bounded linear operator on $L^{2}(0, T ; H)$.

(ii) There exists a constant $K>0$ such that $\sup _{u \in L^{2}(0, T ; G)}\|B(u)(\cdot)\|_{\mathcal{L}} \leq K$, where $\|B(u)(\cdot)\|_{\mathcal{L}}=\sup _{v \in L^{2}(0, T ; H),\|v\|=1}\|B(u)(v)\|_{L^{2}(0, T ; H)}$.

(iii) If a sequence $\left\{u_{k}\right\}_{k=1}^{+\infty} \subset L^{2}(0, T ; G)$ strongly converges to some $u$ in $L^{2}(0, T ; G)$, then, there is a subsequence $\left\{u_{k(l)}\right\}_{l=1}^{+\infty} \subset\left\{u_{k}\right\}_{k=1}^{+\infty}$ such that

$$
B\left(u_{k(l)}\right)^{*}(v) \longrightarrow B(u)^{*}(v) \text { strongly in } L^{2}(0, T ; H)
$$

for any $v \in L^{2}(0, T ; H)$, where $B(u)^{*}(\cdot)$ is the adjoint operator of $B(u)(\cdot)$.

Example. The projection operator $P_{h}(\cdot)$ defined in $(2.3)$ is contained in the class $\mathcal{L}(K)$ in Definition 3.2.

Now, let us recall the abstract convergence theory established in [9].

Proposition 3.3 (Abstract convergence theorem) (cf. [9, Theorem 3.1]). Let $\Psi_{n}$ $(n=1,2, \cdots)$ and $\Psi$ be proper, convex, l.s.c. functionals on $L^{2}(0, T ; H)$. Let $B \in \mathcal{L}(K)$. Assume that $\partial \Psi_{n}$ converges to $\partial \Psi$ in the sense of Graph. Assume that there is a constant $R>0$ so that $u_{n} \in L^{2}(0, T ; H)(n=1,2, \cdots)$ satisfies following conditions;

$$
\left\{\begin{array}{l}
u_{n}^{\prime} \in-B\left(u_{n}\right)\left(\partial \Psi_{n}\left(u_{n}\right) \cap B_{R}\right) \quad \text { in } L^{2}(0, T ; H), \\
u_{n} \in L^{2}(0, T ; G) \\
\left.u_{n}\right|_{t=0}=u_{0, n} \in G
\end{array}\right.
$$

where $B_{R}:=\left\{u \in L^{2}(0, T ; H) ;\|u\|_{L^{2}(0, T ; H)} \leq R\right\}$. If $u_{0, n} \rightarrow u_{0}$ strongly in $H$ and $u_{n} \rightarrow u$ in $C([0, T], H)$ as $n \rightarrow+\infty$, then, the function $u$ is the solution of

$$
\left\{\begin{array}{l}
u^{\prime} \in-B(u)(\partial \Psi(u)) \quad \text { in } L^{2}(0, T ; H), \\
u \in L^{2}(0, T ; G) \\
\left.u\right|_{t=0}=u_{0} \in G
\end{array}\right.
$$

\section{Approximating problem}

In this section we consider the approximating problem of (1.5). At first we shall define the approximating energy function to (2.1). 
For each $\varepsilon>0$, let us define the function $\varphi_{\Delta}^{\varepsilon}$ by the form

$$
\varphi_{\Delta}^{\varepsilon}(u)=\left\{\begin{array}{cc}
\sum_{j<k} c_{j k} \sqrt{\left|a_{j}-a_{k}\right|^{2}+\varepsilon^{2}} & \text { if } u \in H_{\Delta} \\
& \text { with } u(x)=g(x) \text { for } x \in \Gamma, \\
+\infty & \text { otherwise. }
\end{array}\right.
$$

Clearly, $\varphi_{\Delta}^{\varepsilon}$ is proper, l.s.c. and convex on $L^{2}\left(\Omega ; \mathbb{R}^{n}\right)$ such that $\partial \varphi_{\Delta}^{\varepsilon}(\cdot)$ is singlevalued for any $i$ with $\Omega_{i} \cap \Gamma=\emptyset$. More precisely, we have

$$
\partial \varphi_{\Delta}^{\varepsilon}(u)=\sum_{i, j} \frac{c_{i j}}{\left|\Omega_{i}\right|} \frac{a_{i}-a_{j}}{\sqrt{\left|a_{i}-a_{j}\right|^{2}+\varepsilon^{2}}} \chi_{\Omega_{i}} \quad \text { for } \Omega_{i} \text { with } \Omega_{i} \cap \Gamma=\emptyset
$$

for all $u=\sum_{i \in I} a_{i} \chi_{\Omega_{i}} \in H_{\Delta}$, where $\left|\Omega_{i}\right|$ is the volume of $\Omega_{i}$.

Since $\varphi_{\Delta}^{\varepsilon}$ is the approximating function of our energy $\varphi_{\Delta}$ defined by (2.1), the approximating problem to $(2.4)$ is given by the following form

$$
\left\{\begin{array}{lll}
u_{\varepsilon}^{\prime}=-P_{u_{\varepsilon}}\left(\partial \varphi_{\Delta}^{\varepsilon}\left(u_{\varepsilon}\right)\right) & \text { in } L^{2}\left(\Omega ; \mathbb{R}^{n}\right), & \text { a.e. } t \in(0, T), \\
\left.u_{\varepsilon}\right|_{t=0}=u_{0} & \text { in } \Omega .
\end{array}\right.
$$

Here let us mention the result on existence-uniqueness of solutions to (4.3).

Proposition 4.1. Suppose the same condition in Theorem 2.3. Then, for any $\varepsilon>0$ and $T>0$, there exists at most one solution $u_{\varepsilon}$ on $[0, T]$ to the approximating problem (4.3).

Proof. For each given data $u_{0}, g \in H_{\Delta}$ we can prove this Proposition by the slight modification in [10, Subsection 4.3] or [12, Proposition 3.1].

In fact, let $g=\sum_{i \in I} g_{i} \chi_{\Omega_{i}} \in H_{\Delta}$. Then, by taking account of $u_{0}, g \in H_{\Delta}$, (2.3) and (4.2), we observe that the approximating problem (4.3) is reduced to the ODE (ordinary differential equation) system:

$(\mathrm{ODE})$ : Find a unique function $u_{\varepsilon}(t)=\sum_{i \in I} a_{i}(t) \chi_{\Omega_{i}}$ on $[0, \infty)$ such that $a_{i}(t)$ is Lipschitz continuous from $[0, \infty)$ to $S^{n-1}$ satisfying

$$
\begin{array}{r}
a_{i}(t) \equiv g_{i} \quad \text { on } \Omega_{i} \text { with } \Omega_{i} \cap \Gamma \neq \emptyset, \\
\frac{d a_{i}(t)}{d t}=-\pi_{a_{i}(t)}\left(\sum_{j} \frac{c_{i j}}{\left|\Omega_{i}\right|} \frac{a_{i}(t)-a_{j}(t)}{\sqrt{\left|a_{i}(t)-a_{j}(t)\right|^{2}+\varepsilon^{2}}}\right) \\
\text { on } \Omega_{i} \text { with } \Omega_{i} \cap \Gamma=\emptyset,
\end{array}
$$

for each $i \in I$.

Since $a_{i}(t) \in S^{n-1}$ and the projection $\pi_{a_{i}(t)}: \mathbb{R}^{n} \rightarrow T_{a_{i}(t)} S^{n-1}$, we observe that the right hand side of (4.4) is bounded independent of $t$. Hence, by applying the classical theory of ODE (e.g. Cauchy-Lipschitz Theorem), we can get the unique global solution $u_{\varepsilon}$ on $[0, \infty)$ to (ODE), i.e., to our approximating problem (4.3). 


\section{Proof of Theorem 2.3}

In this section, we prove Theorem 2.3 by applying the abstract convergence theory [Proposition 3.3]. We begin with the key lemma to show Theorem 2.3.

Lemma 5.1. Let $\varphi_{\Delta}$ and $\varphi_{\Delta}^{\varepsilon}$ be proper, l.s.c. and convex functions on $L^{2}\left(\Omega ; \mathbb{R}^{n}\right)$ defined in (2.1) and (4.1), respectively. Then, we have:

(i) $\varphi_{\Delta}^{\varepsilon}$ converges to $\varphi_{\Delta}$ on $L^{2}\left(\Omega ; \mathbb{R}^{n}\right)$ in the sense of Mosco [18] as $\varepsilon \rightarrow 0$.

(ii) $\Phi_{\Delta}^{T, \varepsilon}$ converges to $\Phi_{\Delta}^{T}$ on $L^{2}\left(0, T ; L^{2}\left(\Omega ; \mathbb{R}^{n}\right)\right)$ in the sense of Mosco [18] as $\varepsilon \rightarrow 0$, where $\Phi_{\Delta}^{T, \varepsilon}$ is proper, l.s.c. and convex on $L^{2}\left(0, T ; L^{2}\left(\Omega ; \mathbb{R}^{n}\right)\right)$ defined by

$$
\Phi_{\Delta}^{T, \varepsilon}(u)=\int_{0}^{T} \varphi_{\Delta}^{\varepsilon}(u(t)) d t \quad \text { for all } u \in L^{2}\left(0, T ; L^{2}\left(\Omega ; \mathbb{R}^{n}\right)\right) .
$$

Proof. By the general theory of convex analysis and the lower semi-continuity of the total variation, we can easily show (i). The assertion (ii) is the direct consequence of (i).

Proof of Theorem 2.3. By applying the abstract convergence theory [Proposition 3.3 ], we can get the solution of our problem (2.4) as the limit of the function $u_{\varepsilon}$ of (4.3) when $\varepsilon \rightarrow 0$.

Note that the function $u_{\varepsilon}$ is also a solution to the approximating problem

$$
\begin{cases}u_{\varepsilon}^{\prime}=-P_{u_{\varepsilon}}\left(\partial \Phi_{\Delta}^{T, \varepsilon}\left(u_{\varepsilon}\right)\right) & \text { in } L^{2}\left(0, T ; L^{2}\left(\Omega ; \mathbb{R}^{n}\right)\right), \\ \left.u_{\varepsilon}\right|_{t=0}=u_{0} & \text { in } \Omega,\end{cases}
$$

since we observe that $f \in \partial \Phi_{\Delta}^{T, \varepsilon}\left(u_{\varepsilon}\right)$ in $L^{2}\left(0, T ; L^{2}\left(\Omega ; \mathbb{R}^{n}\right)\right)$ if and only if $f(t) \in$ $\partial \varphi_{\Delta}^{\varepsilon}\left(u_{\varepsilon}(t)\right)$ for a.e. $t \in[0, T]$ (for instance, we refer to Brézis [5]).

Now, we take $L^{2}\left(\Omega ; \mathbb{R}^{n}\right)$ as a real Hilbert space $H$, and choose $L^{2}\left(\Omega ; S^{n-1}\right)$ as a non-empty closed subset $G$ in Proposition 3.3. Moreover, from Examples in Section 3 and Lemma 5.1 we observe that the projection operator $P_{h}(\cdot) \in \mathcal{L}(K)$, and $\partial \Phi_{\Delta}^{T, \varepsilon}$ converges to $\partial \Phi_{\Delta}^{T}$ on $L^{2}\left(0, T ; L^{2}\left(\Omega ; \mathbb{R}^{n}\right)\right)$ in the sense of Graph as $\varepsilon \rightarrow 0$.

By the expression (4.2) of $\partial \varphi_{\Delta}^{\varepsilon}\left(u_{\varepsilon}\right)$, we see that the subdifferential $\partial \varphi_{\Delta}^{\varepsilon}\left(u_{\varepsilon}\right)$ is bounded in $L^{2}\left(\Omega ; \mathbb{R}^{n}\right)$ uniformly in $\varepsilon$. Therefore, the subdifferential $\partial \Phi_{\Delta}^{T, \varepsilon}\left(u_{\varepsilon}\right)$ is also bounded in $L^{2}\left(0, T ; L^{2}\left(\Omega ; \mathbb{R}^{n}\right)\right)$ uniformly in $\varepsilon$ for each $T>0$, hence, there is a closed ball $B_{R}$ of $L^{2}\left(0, T ; L^{2}\left(\Omega ; \mathbb{R}^{n}\right)\right)$ such that

$$
\partial \Phi_{\Delta}^{T, \varepsilon}\left(u_{\varepsilon}\right) \subset B_{R} \quad \text { uniformly in } \varepsilon>0 \text { for each } T>0 .
$$

Since $u_{\varepsilon}$ is the solution to $(4.3)$ on $(0, T)$, there is an element $u_{\varepsilon}^{*} \in \partial \varphi_{\Delta}^{\varepsilon}\left(u_{\varepsilon}\right)$ such that $u_{\varepsilon}^{\prime}(\tau, x)=-\pi_{u_{\varepsilon}(\tau, x)}\left(u_{\varepsilon}^{*}(\tau, x)\right)$ for a.e. $(\tau, x) \in(0, T) \times \Omega$. By the definition of $\pi_{u_{\varepsilon}(\tau, x)}(\cdot)$, we see that $u_{\varepsilon}^{\prime}(\tau, x) \in T_{u_{\varepsilon}(\tau, x)} S^{n-1}$ for a.e. $(\tau, x) \in(0, T) \times \Omega$. Thus, we have

$$
\begin{aligned}
\int_{\Omega}\left|u_{\varepsilon}^{\prime}(\tau, x)\right|^{2} d x & =\left(u_{\varepsilon}^{\prime}(\tau),-\pi_{u_{\varepsilon}(\tau)}\left(u_{\varepsilon}^{*}\right)(\tau)\right)_{L^{2}\left(\Omega ; \mathbb{R}^{n}\right)} \\
& =-\left(u_{\varepsilon}^{\prime}(\tau), u_{\varepsilon}^{*}(\tau)\right)_{L^{2}\left(\Omega ; \mathbb{R}^{n}\right)}=-\frac{d}{d \tau} \varphi_{\Delta}^{\varepsilon}\left(u_{\varepsilon}(\tau)\right)
\end{aligned}
$$


for a.e. $\tau \in(0, T)$. By integrating (5.2) over $(0, T)$, we get the energy equation

$$
\int_{0}^{t} \int_{\Omega}\left|u_{\varepsilon}^{\prime}(\tau, x)\right|^{2} d x d \tau+\varphi_{\Delta}^{\varepsilon}\left(u_{\varepsilon}(t)\right)=\varphi_{\Delta}^{\varepsilon}\left(u_{0}\right) \quad \text { for any } t \in[0, T] .
$$

From (5.3) and the compactness theory (cf. [13, Theorem 1.19]) it follows that $\left\{u_{\varepsilon}(t)\right\}$ is relatively compact in $L^{2}\left(\Omega ; \mathbb{R}^{n}\right)$ for any $t \in[0, T]$. Thus, Ascoli-Arzela's theorem implies that there exist a subsequence $\left\{u_{\varepsilon_{m}}\right\}_{m=1}^{+\infty} \subset\left\{u_{\varepsilon}\right\}$ and a function $u \in C\left([0, T] ; L^{2}\left(\Omega ; \mathbb{R}^{n}\right)\right)$ such that $\varepsilon_{m} \rightarrow 0$ and

$$
u_{\varepsilon_{m}} \longrightarrow u \text { strongly in } C\left([0, T] ; L^{2}\left(\Omega ; \mathbb{R}^{n}\right)\right) \quad \text { as } m \rightarrow \infty .
$$

Therefore, since assumptions of the abstract convergence theory [Proposition 3.3] are satisfied, we can apply Proposition 3.3 to our problem. Thus, we conclude that $u$ is the solution on $[0, T]$ to $(2.4)$ (i.e. (1.5)) for each $T>0$.

\section{References}

[1] F. Andreu, C. Ballester, V. Caselles and J.M. Mazón, Minimizing total variation flow, Differential and Integral Equations, 14(2001), 321-360.

[2] F. Andreu, C. Ballester, V. Caselles and J.M. Mazón, The Dirichlet problem for the total variation flow, J. Funct. Anal., 180(2001), 347-403.

[3] H. Attouch, Variational Convergence for Functions and Operators, Pitman Advanced Publishing Program, Boston-London-Melbourne, 1984.

[4] G. Bellettini, M. Novaga and M. Paolini, Facet-breaking for three-dimensional crystals evolving by mean curvature, Interfaces Free Bound., 1(1999), 39-55.

[5] H. Brézis, Opérateurs Maximaux Monotones et Semi-Groupes de Contractions dans les Espaces de Hilbert, North-Holland, Amsterdam, 1973.

[6] F. Catté, P-L. Lions, J-M. Morel and T. Coll, Image selective smoothing and edge detection by nonlinear diffusion, SIAM J. Numer. Anal., 29(1992), 182-193.

[7] L. C. Evans and R. F. Gariepy, Measure Theory and Fine Properties of Functions, Studies in Advanced Mathematics, CRC Press, Boca Raton, FL, 1992.

[8] M.-H. Giga, Y. Giga and R. Kobayashi, Very singular diffusion equations, Proc. Taniguchi Conf. on Math., Advanced Studies in Pure Math., 31(2001), 93-125.

[9] Y. Giga, Y. Kashima and N. Yamazaki, Local solvability of a constrained gradient system of total variation, Abstract and Applied Analysis, 2004(2004), 651-682.

[10] Y. Giga and R. Kobayashi, On constrained equations with singular diffusivity, Methods and Applications of Analysis, 10(2003), 253-278.

[11] Y. Giga and H. Kuroda, On breakdown of solutions of a constrained gradient system of total variation, Bol. Soc. Parana. Mat.(3), 22(2004), 9-20.

[12] Y. Giga, H. Kuroda and N. Yamazaki, An existence result for a discretized constrained gradient system of total variation flow in color image processing, Hokkaido University Preprint Series in Mathmatics $\sharp 734(2005)$.

[13] E. Giusti, Minimal surfaces and functions of bounded variation, Monographs in Mathematics, 80, Birkhuser Verlag, Basel, 1984. 
[14] N. Kenmochi and K. Shirakawa, Stability for a phase field model with the total variation functional as the interfacial energy, Nonlinear Anal., 53(2003), 425-440.

[15] R. Kobayashi and Y. Giga, Equations with singular diffusivity, J. Statist. Phys., 95(1999), 1187-1220.

[16] Y. Kōmura, Nonlinear semi-groups in Hilbert space, J. Math. Soc. Japan, 19(1967), 493-507.

[17] F. Mémoli, G. Sapiro, and S. Osher, Solving variational problems and partial differential equations mapping into general target manifolds, Journal of Computational Physics, 195(2004), 263-292.

[18] U. Mosco, Convergence of convex sets and of solutions variational inequalities, Advances Math., 3(1969), 510-585.

[19] S. Osher, A. Solé and L. Vese, Image decomposition and restoration using total variation minimization and the $H^{-1}$ norm, Multiscale Model. Simul., 1(2003), 349-370.

[20] G. Sapiro, Geometric Partial Differential Equations and Image Analysis, Cambridge University Press, Cambridge, 2001.

[21] K. Shirakawa and M. Kimura, Stability analysis for Allen-Cahn type equation associated with the total variation energy, Nonlinear Anal., 60(2005), 257-282.

[22] B. Tang, G. Sapiro and V. Caselles, Diffusion of general data on non-flat manifolds via harmonic maps theory: The direction diffusion case, Int. J. Computer Vision, 36(2)(2000), 149-161.

[23] B. Tang, G. Sapiro and V. Caselles, Color image enhancement via chromaticity diffusion, IEEE Transactions on Image Processing, 10(2001), 701-707.

[24] A. Visintin, Models of Phase Transitions, Progress in Nonlinear Differential Equations and their Applications 28, Bikhaüser, Boston-Basel-Berlin, 1996.

\section{Acknowledgment}

This work was studied during the stay of the third author at caesar (Bonn) in 2005, and was supported by Ministry of Education, Culture, Sports, Science and Technology, Japan. The third author wishes to thank Professor Karl-Heinz Hoffmann for his valuable comments and kind invitation to caesar in Germany.

Department of Mathematics, Graduate School of Mathematical Sciences, University of Tokyo, 3-8-1 Komaba, Meguro, Tokyo, 153-8914, Japan

E-mail address: labgiga@ms.u-tokyo.ac.jp

Department of Mathematics, Graduate School of Science, Hokkaido University, Kita 10, Nishi 8, Kita-Ku, Sapporo, Hokkaido, 060-0810, Japan

E-mail address: s023012@math.sci.hokudai.ac.jp

Department of Mathematical Science, Common Subject Division, Muroran Institute of Technology, 27-1 Mizumoto-cho, Muroran, 050-8585, Japan

E-mail address: noriaki@mmm.muroran-it.ac.jp 